\title{
Erratum to: A characterization of elementary abelian 2-groups
}

\author{
MARIUS TĂRNĂUCEANU
}

Erratum to: Arch. Math. 102 (2014), 11-14

DOI 10.1007/s00013-013-0581-5

In this short note a correction to our previous paper (Tărnăuceanu in Arch Math 102:11-14, 2014) is made.

1. Introduction. The main result of [2] is Theorem 1.1 which gives a characterization of elementary abelian 2-groups in terms of their maximal (by inclusion) sum-free subsets. Unfortunately, as pointed out by Anabanti [1], it is not true without an additional condition. A correct version is the following.

Theorem 1.1. Let $G$ be a finite group. Then the set of maximal sum-free subsets coincides with the set of complements of maximal subgroups if and only if $G \cong \mathbb{Z}_{2}^{n}$ for some $n \leq 3$.

According to this correction, Corollary 1.2 of [2] will be rewritten in the following manner.

Corollary 1.2. The elementary abelian 2-group $\mathbb{Z}_{2}^{n}$ has $2^{n}-1$ maximal sum-free subsets for $n \leq 3$, and at least $2^{n}$ maximal sum-free subsets for $n \geq 4$.

2. Proof of Theorem 1.1. We can easily check that for $G \cong \mathbb{Z}_{2}^{n}, n \leq 3$, the maximal sum-free subsets coincide with the complements of maximal subgroups.

Conversely, let $M_{1}, M_{2}, \ldots, M_{k}$ be the maximal subgroups of $G$ and assume that $G \backslash M_{i}, i=1,2, \ldots, k$, are the maximal sum-free subsets of $G$. Then $[G$ : $\left.M_{i}\right]=2$, for any $i=1,2, \ldots, k$, by Lemma 2.1 of [2]. We infer that $G$ is a 
nilpotent group, more precisely a 2-group. Since every non-trivial element of $G$ is contained in a maximal sum-free subset of $G$, we have

$$
G \backslash 1=\bigcup_{i=1}^{k} G \backslash M_{i}=G \backslash \bigcap_{i=1}^{k} M_{i}=G \backslash \Phi(G),
$$

that is, $\Phi(G)=1$. Consequently, $G$ is an elementary abelian 2-group, say $G \cong \mathbb{Z}_{2}^{n}$.

Next we will prove that $n \leq 3$. Assume that $n \geq 4$ and denote by $e_{1}, e_{2}, \ldots$, $e_{n}$ the canonical basis of $G$ over $\mathbb{Z}_{2}$. It is clear that $A=\left\{e_{1}, e_{2}, e_{3}, e_{4}, e_{1}+\right.$ $\left.e_{2}+e_{3}+e_{4}\right\}$ is a sum-free subset of $G$. If there is a maximal subgroup $M$ such that $A \subseteq G \backslash M$, then $M$ must be of index 2, which implies that the sum of any two elements of $A$ belongs to $M$. Therefore $e_{1}+e_{2}, e_{3}+e_{4} \in M$ and so $e_{1}+e_{2}+e_{3}+e_{4} \in M$, a contradiction. This completes the proof.

\section{References}

[1] C. Anabanti, A characterization of elementary abelian 3-groups, arXiv:1611.06546.

[2] M. TĂRNĂUCEANU, A characterization of elementary abelian 2-groups, Arch. Math. 102 (2014), 11-14.

MARIUS TĂRNĂUCEANU

Faculty of Mathematics, "Al.I. Cuza" University,

Iaşi,

Romania

e-mail: tarnauc@uaic.ro 